\title{
Berichtigung.
}

In meinem Vortrage auf der diesjährigen Hauptversammlung des Vereins Deutscher Nahrungsmittelchemiker über

\section{Die Anwendung der Feder'schen Verhältniszahl auf Dosenwïste}

(Diese Zeitschrift 1926, 52, 53-60)

ist (S. 55) bezüglich der in Augsburg geltenden Vorschriften über den Wasserzusatz der dortigen Würste eine irrtümliche Auffassung zum Ausdruck gekommen. Von Herrn Dr. S ch mid, Direktor der Städtischen Untersuchungsanstalt in Augsburg, werde ich darauf aufmerksam gemacht, daß auch in Augsburg der zugelassene Fremdwassergehalt, basierend auf der Fe de rschen Zahl 4, sich auf die fertige Wurstware bezieht. Es sind also nicht, wie angegeben, Wasserhöchstzusätze zu den einzelnen Wurstsorten bei der Herstellung vorgeschrieben.

Zur Vermeidung von Mißverständnissen sei dies hiermit festgestellt.

Karlsruhe, den 28. Oktober 1926.

\section{A. Gronover.}

\section{Berichtigung}

zu unserer Arbeit

\section{Über die Giftigkeit des Methylalkohols und dessen Nachweis.}

(Diese Zeitschrift 1926, 52, 117-147.)

In unserer obigen Veröffentlichung führten wir (S. 119) wörtlich aus:

"So schreibt $J$. Ga d a $\mathrm{me}^{1}$ ) dem reinen Methylalkohol kaum toxikologische Bedeutung zu, obwohl er in Dampfform die Schleimhäute reizt und in größeren Mengen Betäubung hervorruft. Die Wirkung des rohen Holzgeistes ist nach ihm eine Kombination der des reinen Methylalkohols und des Acetons (Aceton allein ist ungiftig)."

Hierzu teilt uns Herr Prof. Gadamer mit, daß er inzwischen seine Ansicht geändert habe, und nimmt Bezug auf die 1924 erschienene zweite Auflage seines Lehrbuchs, welche uns bei Abfassung unserer Arbeit nicht zur Verfügung stand. Auf S. 292 dieser Neuauflage heibt es:

„Der reine Methylalkohol soll bei einmaliger Gabe weniger giftig als Äthylalkohol sein. Wiederholte, selbst kleine Gaben, wirken jedoch nachteiliger als Äthylalkohol. Die in den letzten 15 Jahren beobachteten zahlreichen Vergiftungsfälle nach dem Genuß von methylalkoholhaltigem Branntwein sind z. 'T. wohl, nach Theiler sogar ausschließlich - was kaum zutreffen dürfte - auf die Beimengungen, Allylalkohol, Allylacetat, Aceton und dessen sehr giftige Homologe (?), zurückzuführen (Zeitschr. f. angew. Chem. 1923, 148) ${ }^{2}$ ). Jedenfalls aber gehört der Methylalkohol jetzt zu den toxikologisch wichtigsten Körpern. dessen Nachweis den forensischen Chemiker sehr oft beschäftigt."

Diese Ausführungen bringen wir gern zur Kenntnis, ohne uns in allen Teilen mit ihnen einverstanden zu erllären.

E. Dinslage. O. Windhausen.

1) J. Gadamer, Lehrb. d. chem. Toxikologie 1909, S. 298.

2) $\mathrm{Zu}$ einem ähnlichen Ergebnis kommt Euler, C. 21, II, S. 453.

\section{Referate.}

\section{Allgemeine Bestandteile der Lebensmittel.}

0. Loew: Einige Bemerkungen zur Chemie des EiweiBes. (Ber. Deutsch. Chem. Ges. 1925, 58, 2805-2807.) - Verfasser bespricht eingehend die Bildung des Eiweißes in der Pflanze auf Grund der von E. Schulze, Winterstein und Trier erhaltenen Ergebnisse. Das in den Zellen der wachsenden Pflanzenorgane gebildete Eiweiß ist ein äußerst labiler Körper, der ebenso rasch, wie er gebildet wurde, zur Organisation der lebenden Organoide, nämlich Zellkern, Chloroplast. und Cytoplasma verwendet wird; diese können nur dann funktionieren, wenn das ihnen zugrunde liegende Eiweiß sehr labil ist. Es gibt aber Pflanzen, in denen in einer bestimmten Zeit mehr labiles Eiweiß gebildet wird, als zur Bildung der lebenden Organoide nötig ist; dieses häuft sich dann in dem Zellsaft der Vakuole an, wo es sich manchmal in der Form von rundlichen, wasserreichen Schollen oder in Tropfen 